\title{
Aproximación al erotismo diverso y a las realidades LGBTIQ+ contemporáneas en el mundo árabe e islámico
}

An approach to diverse eroticism and contemporary LGTBIQ+ social realities on the Arab and Islamic World

\section{Adrián Rodríguez Iglesias}

Instituto de Lenguas y Culturas del Mediterráneo y Oriente Próximo (ILC-CCHS, CSIC)

adrian.rodriguez@cchs.csic.es

ORCID https://orcid.org/0000-0001-9728-3892 


\section{Resumen}

Este trabajo pretende aportar una perspectiva introductoria en lengua castellana a la consideración y el tratamiento de la diversidad sexual y de género en el mundo árabe e islámico. Así, tras una introducción dedicada a aclarar una serie de conceptos básicos como la arabidad y el hecho islámico, se dedican dos capítulos a tratar el erotismo diverso en la época clásica, trascendiendo el tradicional enfoque dedicado al homoerotismo literario. Posteriormente, se tratan las modificaciones que se produjeron en el Imperio Otomano y que condujeron, más adelante, a la inclusión de la homosexualidad en las legislaciones de los diferentes países de la región. Luego, se realiza un acercamiento a la situación contemporánea en la que el pensamiento islámico interactúa con las corrientes de pensamiento feminista. Por último, se trata la encarnación de las identidades LGTBIQ+ en el mundo árabe, la naciente articulación de movimientos en defensa de los derechos de las personas $L G T B I Q+y$ las representaciones culturales sobre ellas.

Palabras clave: Estudios LGTBIQ+; estudios árabes e islámicos; homoerotismo; islam queer; literatura árabe.

\section{Abstract}

This works is a general and introductory overview on the consideration and treatment of sexual and gender diversity in the Middle-East and North-Africa region (MENA) and among Muslim communities. Thus, firstly, arabicity and the Islamic fact are presented, then two chapters deal with diverse eroticism on Classical Ages, transcending the traditional approach dedicated to literary homoeroticism. Subsequently, we discuss how legislative changes during the Ottoman Empire through the Modern Regime led later the inclusion of homosexuality on the legislations of MENA region countries. Then, we offer an approach to the contemporary situation in which feminism and Islamic thought interacts. Finally, we deal with LGTBIQ+ identities on Arab World, the social reality of these people as well as the emergence of LGTBIQ+ people rights movements and the cultural representations about them on these societies.

Key words: LGTBIQ+ studies; Arabic and Islamic studies; homoeroticism; Queer Islam; Arabic literature. 
Rodríguez-Iglesias, Adrián (2021). Aproximación al erotismo diverso y a las realidades LGBTIQ+ contemporáneas en el mundo árabe e islámico. Revista del Laboratorio Iberoamericano para el Estudio Sociohistórico de las Sexualidades, 06, 63-85-pp. https://doi.org/10.46661/ relies.6130

\section{Introducción}

Este trabajo ofrece una aproximación general al campo de saber que se genera en la intersección de los Estudios Árabes e Islámicos y los Estudios LGTBIQ+ (Lesbianas, Gays, Bisexuales, Transexuales, Intersexuales y Queer). Si bien los estudios sobre la lengua y el mundo árabe en España se desarrollaron, en su origen, fundamentalmente desde el ámbito de la Filología Semítica y de la Historia, paulatinamente han ido integrando, en sus planes de estudio, otras ramas como la Sociología o la Antropología; por otra parte, los Estudios LGTBIQ+ aún son recientes en los planes de estudio de la universidad española y están en pleno auge y desarrollo.

De este modo, se plantea un primer epígrafe introductorio dedicado a la contextualización y exposición de algunos de los conceptos básicos de los Estudios Árabes e Islámicos como son la arabidad y su diferencia con la dimensión religiosa islámica. Los siguientes apartados consisten en una visión panorámica ordenada de forma cronológica sobre la diversidad sexual y de género en el mundo arabo-islámico.

En el tercer apartado, se introduce el erotismo homosexual en la literatura preislámica y clásica, con especial foco en el periodo andalusí, para concluir con nuevas fuentes para el conocimiento de la diversidad sexual y de género que trascienden el ámbito literario, como pueden ser las fuentes legales. En el cuarto, se abordan realidades sexo-genéricas diversas durante los Imperios Islámicos, esto es, aquellos poderes en un marco geográfico no árabe históricamente situados en la Edad Moderna y Contemporánea cuyo poder se ejercía bajo égida islámica.

A continuación, se trata cómo la modernización del marco legal del Imperio Otomano trae consigo una serie de cambios en la legislación sobre las relaciones entre personas del mismo sexo, lo que, junto con la colonización occidental, fundamentalmente británica y francesa, ocasionó una serie de consecuencias que en cierto modo explican la situación legislativa actual.

Los últimos dos apartados se dedican a la Edad Contemporánea: por un lado, a tratar los nuevos enfoques de exégesis islámica que se están dando al conjugarse el pensamiento islámico y la crítica tanto feminista como postfeminista y, por otro, a las realidades LGTBIQ+ en el Mundo Árabe e Islámico y sus representaciones culturales.

El objetivo de este trabajo es ofrecer una panorámica general que sirva de aproximación a este tema, por lo que justificamos algunas ausencias y el tratamiento breve que se ha dado gran parte de las cuestiones, intentado facilitar una bibliografía asequible que permita ahondar en el conocimiento de cada una de ellas.

Por último, debe tenerse en cuenta que los estudios LGTBIQ+ beben en gran parte de la tradición anglosajona y que esta situación se ve también extrapolada al caso concreto del mundo áraboislámico cuando se trata de producción de conocimiento dentro del ámbito de los estudios LGTBIQ+. Por ello, y aunque esto plantee ciertas limitaciones, pues fundamentalmente supone un reforzamiento del etnocentrismo generalizado en los análisis de género y sexualidad que tratan este contexto sociocultural y geográfico, no debe extrañar que la mayoría de la bibliografía referida en este trabajo sea también anglosajona. Con todo, esta bibliografía suele ser también la más asequible 
para el mundo hispanófono para el que se redacta este artículo cuya finalidad, como se ha dicho, es principalmente introductoria.

\section{Lo árabe, lo islámico y lo musulmán}

Es necesario aclarar en primer lugar algunos conceptos clave en los estudios árabes e islámicos para establecer las convenientes categorías.

En primer lugar, se debe tener en cuenta que el término "árabe" es susceptible de designar, al menos, dos conceptos: uno sociocultural y otro lingüístico. Desde un punto de vista sociocultural, la expresión "pueblos árabes" hace referencia a aquellos pueblos que habitan la región geográfica de la Península Arábiga, incluidas Siria y Jordania, independientemente de su religión, por lo que se incluye en ella a personas de distintas religiones, como pueden ser musulmanes y cristianos.

Sin embargo, entre los siglos VII y VIII tras la predicación de Mahoma, estos pueblos llevan a cabo una expansión geográfica, las llamadas "conquistas árabes". En estos territorios, que hoy en día conforman el Oriente Próximo hasta Irak y todo el Norte de África, la lengua árabe se convirtió en la lengua vehicular y mayoritaria mediante el proceso de arabización.

El concepto de "arabidad" se expande así y refiere a aquella población cuya lengua materna es alguna de las variedades del árabe dialectal. Esto conlleva varias consecuencias: que el concepto lingüístico prime sobre el sociocultural y que el área geográfica a la que se aplique exceda con mucho el área geográfica original de la Península Arábiga. Actualmente, la Liga de los Estados Árabes está conformada por veintitrés países ${ }^{1}$.

En segundo lugar, la revelación de la fe musulmana a Mahoma, que consagra la lengua árabe como lengua litúrgica del islam, junto con una serie de factores sociales como la apuesta por la solidaridad entre tribus del desierto y una serie de reformas económicas y sociales permiten que, durante la vida de Mahoma, se unifique el mandato sobre el territorio de la Península Arábiga bajo su égida. A su muerte en el 632 d. C., tras una serie de enfrentamientos por la sucesión, comienza el período en el que las dinastías omeya y abasí llevaran a cabo estas conquistas árabes, también conocidas como "expansión musulmana". La fe musulmana se expande, a la par que la lengua árabe, en un proceso de islamización que se realiza en paralelo al de arabización.

Sin embargo, los territorios donde la fe musulmana se hará mayoritaria desbordarán posteriormente las fronteras del mundo árabe. Estos territorios donde la fe musulmana es mayoritaria es lo que solemos referir como mundo islámico, en árabe dār al-Is/ām. En la actualidad, el islam es la segunda religión con más fieles en el mundo y es mayoritaria en gran parte de los países de África Occidental y Asia Central incluyendo zonas del subcontinente indio como Pakistán y Bangladés, así como en Oceanía, siendo Indonesia el país donde con mayor número de fieles. También goza de presencia en Europa, desde época otomana en países como Albania o BosniaHerzegovina y en otros desde época más reciente como Francia, donde la población musulmana constituye entorno al $10 \%$ de la población.

\footnotetext{
${ }^{1}$ Estos países son Egipto, Irak, Jordania, Líbano, Arabia Saudita, Siria, Yemen, Libia, Sudán, Marruecos, Túnez, Kuwait, Argelia, Emiratos Árabes Unidos, Baréin, Catar, Omán, Mauritania, Somalia, Palestina, Yibuti y Comoras. La República Árabe Saharaui Democrática, antigua colonia española del Sáhara Occidental, no forma parte de la Liga, ya que Marruecos no reconoce esta autoridad, aunque esta decisión no es apoyada por Mauritania y Argelia. Por otra parte, Chad, pese a reconocer el árabe como idioma oficial y poseer mayoría de población musulmana, no forma parte de la Liga.
}

Revista del Laboratorio Iberoamericano para el Estudio https://doi.org/10.46661/relies.6130 
Por tanto, antes de abordar cualquier estudio en relación con el mundo árabe e islámico es necesario diferenciar entre lo árabe -concepto en origen étnico, pero fundamentalmente referido a lo lingüístico- frente a lo islámico - un fenómeno religioso-.

El islam, desde la muerte de Mahoma, sufre una serie de cismas que hacen que se divida en tres grandes grupos: sunnitas, chiítas y jariŷíes. Pese a que el inicio de estos cismas se fundamenta en quién debía detentar el poder sucesorio, esta división también supuso una diferente interpretación y desarrollo de las ciencias teológicas y, en consecuencia, también de las ciencias jurídicas. De este modo, en torno a la jurisprudencia se desarrollarán aún más divisiones en diferentes escuelas dentro de cada rama del islam. En la actualidad, el islam sunní es mayoritario a nivel global, mientras que el chií es mayoritario en Irak y, fundamentalmente, en Irán y los ibadíes, una de las ramas jariŷíes, son mayoritarios en el sultanato de Omán.

Las relaciones entre el islam y la política son mucho más amplias. Aunque precisamente la religión se incorpora en todos los ámbitos de la vida musulmana y su organización social, es necesario hacer mención a la polisemia que en nuestra lengua castellana tiene la palabra "islamismo" 2 . Su segunda acepción es la que motiva el adjetivo "islamista", que no equivale ni a "islámico" ni a "musulmán", aunque pueda haber musulmanes islamistas, es decir, seguidores de los diferentes movimientos islamistas ${ }^{3}$, como puede ser el wahhabismo ${ }^{4}$.

Del mismo modo, debe también tenerse en cuenta que el impacto del componente religioso, lo islámico, trasciende este ámbito y se imbrica en todos los ámbitos de la vida del creyente, lo que deriva en un constructo que permea y cristaliza de manera muy evidente en lo cultural, lo político y lo identitario, emergiendo así el concepto de musulmaneidad.

Por último, empleamos en este artículo Occidente como término paraguas para referir las culturas nacidas en Europa bajo el pensamiento cristiano, de herencia grecorromana, extendida hoy a otros ámbitos geográficos del Norteamérica y Oceanía por efecto de la colonización. Del mismo modo, denominamos Modernidad al régimen socio-político que surge, a partir del siglo XV y tiene su cumbre programática en la llustración, que se asienta sobre un programa cultural basado en el imperio de la ley, la secularización del estado y un régimen de derechos y deberes de la ciudadanía.

Sin embargo, más allá del régimen sociopolítico y su curso histórico en los países occidentales y sus consecuencias de la que abunda literatura académica, en este artículo hay que destacar el gran impacto de este régimen en la producción de saber sobre otras realidades culturales e históricas. El resultado es, muy a menudo, un conocimiento etnocéntrico y colonial cuando se genera en relación

\footnotetext{
${ }^{2}$ El Diccionario de la Lengua Española de la Real Academia Española en su 23a edición considera dos acepciones: una, que designa "el conjunto de creencias y preceptos morales que constituyen la religión de Mahoma", y otra, más reciente, que refiere la "ideología que subyace al fundamentalismo islámico".

${ }^{3}$ Los movimientos islamistas, fundados a raíz de las diferentes reformas de pensamiento político fundamentalmente en el s. XVIII y que se implementaron en ciertos territorios en el s. XX, entienden que la formulación del Estado moderno debe beber directamente de las fuentes de derecho islámicas, como en la República Islámica de Irán, tras la revolución de 1979, o como ha ocurrido en algunas zonas de Siria e Irak durante la pasada década.

${ }^{4}$ Corriente política y religiosa sunní de escuela hanbalí, profesada de forma estatal en Arabia Saudí, que persigue volver a la pureza del islam, entendida esta como su estado original tal y como fue revelado. Dentro del Islam, la ortodoxia y el fundamentalismo de esta corriente causa bastante controversia. Así, algunos de los preceptos y actitudes que adoptan, como la iconoclasia llevado a cabo desde el s. XVIII hasta actualidad que, por ejemplo, ha conllevado la destrucción paulatina del patrimonio y algunos los lugares asociados a los inicios de la religión (enterramientos, casas de personas cercanas al profeta, tumbas, ...) o la consideración de que la celebración de fiestas de santos va en contra de la tradición islámica, circunstancias que son, por el contrario, costumbres muy arraigadas en el islam de ciertos territorios como el Norte de África, donde es frecuente la veneración de santos y la conservación de sus mausoleos.
} 
a aquellas regiones y culturas que, como el caso del mundo árabe e islámico, han sido tratadas en relación de alteridad.

\section{Erotismo diverso en la literatura árabe preislamica y clasica}

La periodización de la Historia árabe en relación a la establecida por Europa difiere en cuanto la segmentación en periodos históricos. Como se ha visto, la revelación del islam es el hecho fundacional de la civilización y la hégira, la huida de la primigenia comunidad musulmana de La Meca a Medina en 622, inicia el calendario musulmán. El periodo histórico anterior se conoce como

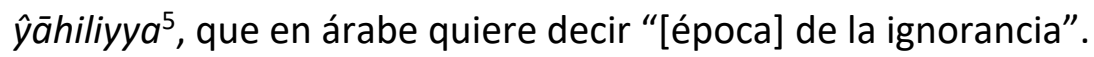

En cuanto a la literatura árabe, la primera gran obra escrita en lengua árabe es el Corán que, para la fe musulmana, es palabra de Alá revelada a Mahoma. La situación lingüística anterior apenas se conoce por la escasez de registros que se conservan de entre los siglos III y VIII, y en los que se representa un haz de dialectos semíticos (Ferrando, 2001; Vicente, 2008).

El conocimiento de la poesía preislámica se reduce en gran medida a las composiciones líricas denominadas mu'allaqāt (trad.: Corriente y Monferrer, 2005), "las colgadas". Se trata de composiciones monorrimas que se bordaban en oro y se colgaban en una tela negra sobre la roca de la Kaa'ba en Meca tras una especie de juegos poéticos. Las conocemos a través de fuentes indirectas: las recopilaciones hechas posteriormente por autores musulmanes en el siglo X.

Con todo, tras el estudio de estas composiciones se ha apreciado, entre otras cosas, el arquetipo social del caballero nómada, beduino, marginado y fuera de la ley, que posee un rígido código de honor. La crítica y los estudios literarios señalan, además, el erotismo presente en esta lírica (Veglinson, 1997) y la sanción de los compiladores (Rowson, 2006) de la sociedad preislámica donde tenían cabida la prostitución y el homoerotismo. Es más, en este medio sociocultural preislámico, la existencia de identidades y prácticas no binarias parece, de acuerdo de nuevo con Rowson (1991) atestiguada con la figura de mujannat, asimilado, como sucederá en otros contextos que veremos posteriormente, a la figura de bailarín, copero o cantor travestido o afeminado.

En oposición al periodo anterior de la ignorancia (del islam), se sitúa el tār'īj, es decir, la Historia. Esta periodización árabe de la historia suele distinguir un largo periodo que abarca califatos e imperios hasta la primera mitad del XIX, que es cuando se produce la Nahḍ, el "Renacimiento". Sin embargo, lo que se conoce como "época dorada de la civilización islámica" se suele referir a los califatos omeya, con capital en Damasco entre los siglos VI y VIII y que tiene una prolongación en la Córdoba andalusí durante el siglo X, y abasí, con capital en Bagdad entre los siglos VIII y XIII.

En cuanto la literatura de esta época, la poesía árabe clásica ${ }^{6}$, lo más significativo en lo relativo a estudios de género y diversidad sexual es el establecimiento de los cánones clásicos del amor en los siglos VII y VIII: el amor 'udrí, caracterizado por tratar un amor casto y puro que, para algunos autores, preconiza el canon del amor cortés medieval en Europa, frente al amor ibāhī, el amor sensual.

\footnotetext{
${ }^{5}$ El sistema de transliteración del árabe es el de la Escuela de Arabistas Españoles.

${ }^{6}$ Algunas de las referencias generales para este tema en castellano son: Veglinson Elías de Molins, Josefina. (1997). La poesía árabe clásica. Madrid: Hiperión; Rubiera Mata, María Jesús. (1999), La literatura árabe clásica: desde la época pre-islámica al Imperio Otomano. Alicante: Universidad de Alicante; Sobh, Mahmud. (2002) Historia de la Literatura árabe clásica. Madrid: Cátedra. En concreto sobre poesía andalusí: Rubiera Mata, María José. (1992). Literatura hispanoárabe. Madrid: Mapfre.
}

Revista del Laboratorio Iberoamericano para el Estudio https://doi.org/10.46661/relies.6130

Sociohistórico de las Sexualidades 


\subsection{Homoerotismo en la literatura árabe clásica ${ }^{7}$}

Las composiciones líricas con pasajes homoeróticos aparecen a menudo en la poesía báquica, satírica y amorosa de esta literatura árabe clásica y se han dedicado estudios monográficos a este tema (Rowson y Wright, 1997).

Entre los autores orientales, por destacar una figura de esta época, merece ser nombrado el abasí Abū Nuwās (s. VIII) escritor muy prolífico que cultivó géneros como las jamriyāt, composiciones dedicadas al vino o, también mudakkarāt, composiciones dedicadas a los hombres, con figuras retóricas que a menudo describen escenas de sexo explícito entre hombres. En este sentido, quizá sea necesario aclarar que el concepto de "género" en la literatura árabes clásica no corresponde al actual género literario, sino más bien al concepto actual de tema. Y es más, el desarrollo de este tipo de composiciones por parte de este autor llega a tal punto que inaugura el género de las gumlāmiyyat, dedicadas a gulām, los "imberbes" o efebos.

Sin embargo, estas composiciones no solo se dan en el Oriente sino que se cultivan, por ejemplo, frecuentemente en al-Ándalus. Se conocen autores de este tipo de composiciones de toda condición social desde época omeya como el emir Abd Allah I (844-912), Ibn 'Abd Rabbihi (860-940), Ibn Sa 'dūn (927-972) o la princesa omeya Wallada. En épocas posteriores al califato omeya de Córdoba, tampoco faltan testimonios y, en época de taifas, componen autores como Ibn Šuhayd (992-1035), Ibn Quzman, el rey de Sevilla al-Mu'tamid (1040-1095), Ibn 'Ammar (1031-1086) en árabe, lo que también ocurría en lengua hebra con autores como Samuel bin Nagrella (993-1095), Ibn Gabirol (1021-1055), Mošeh ibn Ezra (1055-1138) o Yahudah Halevi (1074-1141). Es necesario añadir que la práctica totalidad de estas composiciones se hallan en recopilaciones posteriores, como puede ser el Rāyāt al-mubarrizinn wa-gāyāt al-mumayyizin (El libro de las banderas de los campeones) (trad. García Gómez, E., 1978], de Ibn Sa īd al-Magribī, autor alcalaíno del siglo XIII.

Del mismo modo, el homoerotismo femenino también aparece en la literatura árabe clásica (MaltiDouglas, 2001; Habib, 2009, 2012), siendo el testimonio homoerótico femenino más temprano que se conserva es la historia de amor intercultural e interreligioso de finales del siglo VI que tuvieron Hind bint al-Nu'mān, princesa rey lajmí del reino de Hira ${ }^{8}$ y Hind bint al-Juss al-lyādiya, árabe, apodada al-Zarqa', cuya historicidad ha sido puesto en duda. Sin embargo, su amor, así como el de muchas otras, lo conocemos por referencias en obras varios siglos posteriores diferentes de autores y con diferentes fines como pudieron ser el Kitāb al-Agānī (Libro de las canciones) de al-Isfahani o en el Ŷawāmi' al-Laḍda (La enciclopedia del placer) de 'Alī ibn 'Alī al-Kātibī ambos autores del siglo $X$, siendo estas referencias, muy a menudo, el único vestigio conservado (Amer, 2009).

\footnotetext{
${ }^{7}$ La periodización árabe de la Historia tendría por "literatura clásica” aquella producida hasta el Renacimiento árabe del s. XIX. Sin embargo, habitualmente esta etiqueta para los estudios árabes e islámicos suele referir la considerada "época de oro del islam", que coincide con la expansión islámica, es decir, el periodo comprendido entre los ss. VIII-XV, que casi coincide con la Edad Media Europea.

${ }^{8}$ Reino preislámico que ocupó el norte de la Península Arábiga y el oeste del actual Iraq desde el siglo III hasta su conquista por las tropas islámicas en el siglo VII, que fue gobernado por los lajmidas, árabes cristianos nestorianos mesopotámico, cuya capital estaba en la ciudad Hira (actual Iraq).
}

Revista del Laboratorio Iberoamericano para el Estudio

Sociohistórico de las Sexualidades 


\subsection{Más allá del homoerotismo en la literatura árabe clásica: concepción de género, naturaleza del amor y jurisprudencia sobre intergenitalidad}

Las figuras retóricas, más o menos explícitas, que se encuentran en la literatura y refieren a lo que actualmente denominamos diversidad sexual no son la única fuente donde se tratan estos temas. En este apartado presentamos tres casos de interés en este ámbito como son un manual de erótica, una obra de mística y, por último, fuentes legales.

En primer lugar, el tratado de erótica Țawq al-Hamāmat, conocido como El Collar de la Paloma escrito por Ibn Hazm de Córdoba en el s. X da buena cuenta del ars amandi de la época, un tratado sobre amatoria bajo la égida del canon del amor platónico. Así, entre los ejemplos que aporta sobre concepción amatoria, también incluye una reflexión sobre la naturaleza del amor y una exposición de casos que el autor considera raros. Sin embargo, entre ellos, parece reconocerse un caso que, convenimos en recoger bajo la categoría de aerotismo (Rodríguez Iglesias, 2019), en analogía con el homoerotismo, y que sería un testimonio precedente de la moderna identidad asexual.

Dentro del molde neoplatónico que circula en los patrones de esta época, la concepción del alma que se manifiesta en las composiciones y obras sobre mística también arroja nuevas perspectivas. Es el caso por ejemplo de Ibn 'Arabí de Murcia (Murcia, 1165 - Damasco, 1240), quien en varias de sus obras afirma que la división sexual en hombre o mujer no son más que meros accidentes que no son esenciales a la noción de humanidad. Evidentemente, esto se contextualiza, dentro de su pensamiento filosófico ${ }^{9}$, con su postura sobre la posibilidad de desarrollarse como al-insān al-kāmil (ár. el ser humano completo, perfecto). Si bien, con ello, trasciende la idea platónica de que las mujeres no tienen alma y gran parte de los patrones de género que se dan habitualmente por supuesto quedan en sus obras subvertido.

Por último, las fuentes para el estudio de la diversidad sexual no solo se circunscriben a las fuentes literarias. Así, el estudio de sobre la legislación medieval, fundamentalmente chií del siglo XIV, arroja luz de un fenómeno, denominado junța muškil, es decir, "el problema de ser como mujer" (Sanders, 1991), que no son otra cosa que disposiciones legales para determinar con quien debe haber matrimonio o el lugar que deben ocupar en la mezquita, entre otros aspectos, aquellas personas cuyos genitales sean ambiguos, esto es, de lo que hoy denominamos como personas en situación de intergenitalidad.

\section{De la baja edad media al siglo xix: el poder otomano y los imperios islámicos modernos}

La periodización árabe de la historia no divide, como se indicó previamente, este periodo del anterior. Si bien es cierto que la organización del mundo árabe cambia radicalmente entre el periodo que en este epígrafe nos ocupa, el que se comprende entre los siglos XIII y XIX. EI mundo árabe deja atrás la que, a menudo, es Ilamada la Edad de Oro del islam (ss. VIII - XIII) en la que se produce la expansión geográfica y su poder político que abarca en el siglo XIII desde al-Ándalus, en un extremo, hasta Afganistán, en el otro. Con esta expansión sucede también la de la religión islámica y se conforma una cultura árabe de gran calidad e impacto en el resto del mundo con grandes avances

\footnotetext{
${ }^{9}$ Como referencia general al pensamiento filosófico de este autor, el capítulo "Ibn 'Arabi y su escuela" dentro de la obra clásica de Henry Corbin de 1964, Historia de la Filosofía Islámica (Corbin, [2a ed. trad.] 2000: 563-571), aunque tampoco faltan estudios monográficos sobre lo femenino en Ibn Arabi como el de López Anguita, G. (2010) “El principio femenino en los textos cosmológicos del sufí Mujyiddin Ibn Arabí (1165 - 1240)" en Torres, K. (ed.) La mujer musulmana desde la traducción a la realidad. Sevilla: Arcibel; Murata, S. (1992) The Tao of Islam. A Sourcebook on Gender Relationships in Islamic Thought. Nueva York: New York University Press.
}

Revista del Laboratorio Iberoamericano para el Estudio https://doi.org/10.46661/relies.6130

Sociohistórico de las Sexualidades 
en ciencia, medicina, agricultura, comercio, etc., situación que contrasta claramente con la que se produce durante la Alta Edad Media en Europa tras el colapso del Imperio Romano.

Sin embargo, a mediados del s. XIII, el califato abasí es conquistado por los mongoles y la institución que gobernaba estos territorios colapsa y se sucede una época convulsa con diferentes poderes de ámbito geográfico más reducido. Sin embargo, en ese mismo siglo se fundará en Anatolia una pequeña entidad política que estará llamada a construir la hegemonía en la región hasta el s. XIX, el imperio otomano.

Por otro lado, durante este periodo y fundamentalmente más entrada la Edad Moderna el islam se extenderá a territorios fuera del mundo árabe, en Asia Central, África, así como en el subcontinente indio e incluso el Lejano Oriente. En este periodo se fundarán imperios en Asia Central y el subcontinente indio cuya religión de estado será el islam, produciéndose un interesante dialogismo entre las instituciones previas autóctonas y la configuración de estas nuevas sociedades.

\subsection{El Imperio Otomano hasta el s. XVIII}

Los orígenes del Imperio Otomano se remontan a 1299, en ese entonces su territorio ocupa solamente un pequeño principado situado al suroeste del actual Estambul, que irá paulatinamente conquistando territorios al Imperio Bizantino tanto en Anatolia como en los Balcanes. No será hasta la conquista de Constantinopla en 1543, desde entonces Estambul, cuando empiece su mayor auge que tendrá lugar en el s. XVI y será cuando alcance su mayor extensión, controlando gran parte del Norte de África, del Oriente Próximo y los Balcanes.

En cuanto a la diversidad sexual y de género en este periodo histórico, hay estudios sobre la situación en la Edad Media, con especial foco en los eunucos, de variada tipología y función social o el concubinato (El-Azhari, 2019), así como la homosexualidad en el espacio temporal que transcurre hasta el final de la Edad Moderna (El-Rouayheb, 2005).

Asimismo, durante sobre el periodo previo a su expansión por el Norte de África, existen trabajos sobre cuestiones relacionadas con la diversidad sexual y de género en territorios que, posteriormente, quedarán incluidos en él, como, por ejemplo, Egipto, donde el poder otomano se impone en el siglo XVI ${ }^{10}$.

El sultanato mameluco gobierna este territorio desde 1250 hasta su conquista por los otomanos en 1517. Durante la época mameluca, el homoerotismo aparece también en la literatura (Murray, 1997a, en español existen varios trabajos monográficos: Shafik, 2020; Lucena Romero 2021).

La vasta extensión del imperio dará lugar a una sociedad cosmopolita, pero el aumento poblacional que conlleva su expansión tendrá una contrapartida, un debilitamiento del poder central. De este modo, a partir de mediados del s. XVI, el Imperio Otomano entra en una etapa de decadencia, favorecida por la desmembración del ejército hasta entonces conformado fundamentalmente por el cuerpo de jenízaros, soldados profesionales obligados a mantenerse célibes, así como por una serie de sultanes que sucumben a las intrigas palaciegas y a la corrupción instaurada en la administración.

\footnotetext{
${ }^{10}$ Hasta entonces, Egipto había sido independiente del califato abasí de Bagdad desde mediados del siglo IX bajo la dinastía tuluní y se convirtió, posteriormente, en el epicentro del califato fatimí tras la fundación de El Cairo en 969 . El califato fatimí, de rito chií y fundado en Mahdía (Túnez) en 916 d.C., ocupaba el Norte de África y sucumbió ante dos entidades políticas: los almohades, en la región occidental, y los ayubíes, en la región oriental. La dinastía ayubí, fundada por Saladino, integra, de nuevo, a Egipto en la órbita del califato abasí, ya que los ayubíes gozaban de cierta autonomía. Con todo, Egipto será el último reducto de este califato abasí, cuando Bagdad sea conquistada por los mongoles en 1257 , pero será gobernado por otro régimen, el sultanato mameluco.
}

Revista del Laboratorio Iberoamericano para el Estudio

Sociohistórico de las Sexualidades https://doi.org/10.46661/relies.6130 
Esta conjunción de factores favorece que los gobernantes locales de los diferentes territorios obtengan un mayor grado de autonomía y en otros la pérdida del control y poder sobre los mismos. El primer caso será el sistema conocido como beylicato, que favorecerá la independencia a lo largo del XVIII y XIX de algunos territorios, como, por ejemplo, ocurre durante el Beylicato de Túnez (17051881). La reforma y el restablecimiento del poder político otomano se llevará a cabo en los siglos XVIII y XIX y será tratado, en detalle, en el capítulo quinto.

\subsection{Otras realidades de diversidad de género y sexualidad no normativa en los imperios islámicos modernos}

Como se indicó previamente, tras las conquistas islámicas, la fe musulmana se expande más allá de las fronteras del mundo árabe. La intersección entre las culturas nativas y la dominación musulmana deja entrever una cierta permisibilidad del islam hacia realidades de género no binario en otros territorios, fuera del contexto árabe, donde se impone un poder musulmán. Ilustramos aquí esta siuación con tres casos: hijra en India, burrnesh en Albania y bacha bazi en Afganistán.

El subcontinente indio, tiene dos periodos de dominación musulmana: el sultanato de Delhi (ss. XIII$\mathrm{XVI}$ ) y el imperio mogol (s. XVI-XIX), durante los cuales parece que estas personas gozaban de aceptación social, podían poseer bienes y desempeñar ualquier cargo. De este modo, lyer esboza la relación entre hijra y la sociedad islámica moderna: "The word hijra is believed to be an Urdu term and suggests that hijra culture and identity evolved during the Delhi Sultanate and the Mughal Empire in India where eunuchs served as confidants, political advisors, and guardians of the harem" (lyer, 2009: 241).

Es más, en relación al presente artículo, es de interés indicar que, como recoge la autora: "hijras identify themselves as Islamic and many perform the hajj to Mecca and assume Muslim names. They also worship at the tombs of Muslim mystics along with the other Indian Muslims and celebrate Islamic holidays. However, hijras also mantain Hindu practices" (lyer, 2009: 241). Sin embargo, también hay otros estudios que sugieren que es posible que, en ciertos momentos históricos y también bajo la égida islámica, hubiera temor ante estas personas y diferencias en cuanto a su tratamiento y consideración al no encajar en el patrón binario islámico (Mposo, 2017: 50-51).

La llegada de la colonización británica, periodo conocido como Rāj (1848 - 1947), impone el paradigma binario de género y condena a la exclusión social a quien está fuera de él, lo que está en relación con el mundo de la prostitución. Con la Independencia del Reino Unido (1947) y la instauración de la actual República de India (1950), la situación tardó en mejorar, ya que el reconocimiento legal de hijra no llegó hasta 2014, así como la despenalización de la homosexualidad en 2018.

Otra realidad que conlleva un cambio en los roles e incluso la expresión de género se encuentra en Albania ${ }^{11}$, donde burrnesh, también llamadas virgjeneshtë o "vírgenes juradas" son mujeres que juran mantener su virginidad ante la comunidad $y$, por ello, pueden ejercer en sociedad como varones. Es decir, el celibato se pone como condición para poder realizar un cambio en los roles de género que, además, también suele ir asociada por una estética más masculinizada. Esta forma de vida de las "vírgenes juradas" parece haberse mantenido, aunque la mayoría de noticias sobre ellas provenga de descripciones antropológicas del s. XX, si bien es cierto que recientemente han sido

\footnotetext{
${ }^{11}$ La relación de este país balcánico con el mundo arabo-islámico viene dada porque entre los siglos XIV y XX formo parte del Imperio Otomano, del que se independizó en 1912. Es más, los procesos de islamización de Albania que tuvieron lugar fundamentalmente entre los siglos XVII y XVIII triunfaron de tal manera que hoy en día la religión mayoritaria entre la población albanesa sigue siendo el islam.
}

Revista del Laboratorio Iberoamericano para el Estudio

Sociohistórico de las Sexualidades https://doi.org/10.46661/relies.6130 
objeto de varios estudios (Grémaux, 1996; Dickemann, 1997; Mislevska, 2005, etc.). Actualmente, son una forma de vida en desaparición.

En otro orden, debe tenerse en cuenta prácticas de sexualidad no normativa incluidas en este apartado por tratarse de prácticas entre personas del mismo sexo. Este es el caso, por ejemplo, de lo bacha bazi, los "niños cantores", una realidad aún presente en algunas zonas de Asia Central, que entronca con la figura del Ganimedes griego. Se trata de menores que bailan y cantan para otros hombres y, a menudo, son tratados como esclavos, ya que representan un símbolo de estatus y poder. El mundo occidental conoció esta realidad a través de la literatura de viajes del XIX, en este caso concreto de Eugene Schuyler (1876: 132-3), así como a través de los trabajos etnográficos de fotografía que llevó a cabo la administración rusa al tomar el control de esta parte de Asia Central a mediados del XIX, como por ejemplo algunas fotografías de grupos de bacha bazi que se encuentran en el Turkestan Album comisionado por Konstantin Petrovich von Kaufman $(1818-1882)^{12}$.

En la actualidad aún se conservan en ciertas en áreas de Afganistán y el noroeste de Pakistán como pueden atestiguar varios trabajos de campo realizados recientemente (por ejemplo, desde un enfoque antropológico y de la salud: Lind van Wijngaarden, J.W. y Rani, B. 2011, o desde una perspectiva legalista: Jones, S. V., 2015). La situación actual, pese a ser una práctica penada tanto por el islam como por la legislación afgana, aparece en la ficción literaria, por ejemplo, en la novela de Khaled Hosseini, Cometas en el cielo (2003, [trad. 2004]) y, del mismo modo, también atrajo la atención de documentaristas, como se mostró, entre otros, en el documental The Dancing Boys of Afghanistan (Quraishi, 2010).

\section{De la modernizacion del imperio otomano a la legislacion contemporanea}

A mediados del siglo XIX, los sultanos otomanos llevan a cabo una serie de reformas en el seno de la administración imperial que obedecen a un intento de modernización y secularización siguiendo los postulados de la llustración europea. El interés en este periodo concreto de la historia del Imperio Otomano trasciende el hecho de las manifestaciones concretas que permiten trazar una historia social y obedece, más bien, al cambio sustancial de régimen. En este sentido, los cambios y reformas que se introducen repercuten en el trato y consideración que reciben las relaciones entre personas del mismo sexo, como se verá con más detalle en el primer epígrafe de este apartado. En consecuencia, la inclusión o no de la homosexualidad en los códigos penales de los diferentes países árabes y las vías para justificar su inclusión en ellos ocupan el segundo apartado, mientras que en el tercero se ofrece una panorámica general de la situación actual sobre la legislación sobre la homosexualidad.

\subsection{El tránsito hacia la Modernidad: el periodo de Tanẓimāt (1839 - 1876) y sus consecuencias en el área de influencia otomana.}

El Imperio Otomano fue un estado multiétnico y multi-confesional, que ocupó gran parte de los territorios de Europa Oriental, Norte de África y Oriente Próximo. Tras el periodo de esplendor, el Imperio entra en decadencia a lo largo de los s. XVII y XVIII, un periodo de fragmentación y concesión de autonomía a la mayoría de territorios que lo conformaban.

A mediados del s. XIX se inicia un proceso de reforma de su estructura imperial que funda las bases del Estado otomano moderno: es el periodo conocido como Tanżimāt (1839 - 1876). El objetivo es modernizar y secularizar el Estado. De este modo, entre otras medidas, se adopta el Código

\footnotetext{
${ }^{12}$ Disponible el álbum en la World Digital Library: https://www.wdl.org/en/item/10829/

Revista del Laboratorio Iberoamericano para el Estudio Sociohistórico de las Sexualidades https://doi.org/10.46661/relies.6130
} 
Napoleónico de Comercio (1850), se lleva a cabo la abolición de impuesto de capitación para no musulmanes y, posteriormente, se adopta el Código Penal Francés (1858).

A partir de entonces se sucede una serie de cambios fundamentales: por una parte, la asunción del modelo de Estado Moderno que, en Occidente, conlleva la paulatina distinción y disociación entre pecado y delito; por otra, una reorganización de la jurisprudencia religiosa y los marcos de poder moral de la cultura islámica a esta nueva forma de organización estatal. La adopción del marco legal napoleónico, en concreto del Código Penal Francés, que no contemplaba las relaciones entre personas del mismo sexo, supone que se replique esta situación en el Imperio Otomano, es decir, a partir de 1858 las relaciones sexuales entre personas del mismo sexo, a priori, quedan despenalizadas en arreglo a un vacío legal. Del mismo modo, entre los siglos XVI y XVIII desde los estudios culturales se ha tratado el cambio que produce en el discurso sexual (Ze'vi, 2006) y también existen monográficos sobre esta época, por ejemplo, sobre la homosexualidad en el siglo XVIII (Muray, 2007) o sobre los músicos profesionales afeminados de acuerdo con las fuentes de la poesía cortesana otomana y la música de los siglos XVIII y XIX (Klebe, 2005).

El Imperio Otomano será derrocado en 1922. Kemal Atatürk instaura la República de Turquía en 1923, siguiendo el modelo europeo moderno de república laica como referencia.

\subsection{Legislación contemporánea sobre orientación sexual}

La legislación sobre la homosexualidad en el mundo islámico puede tener dos bases jurídicas: la doctrina legal o la doctrina religiosa. En cuanto la doctrina legal, la introducción de las prácticas sexuales entre dos personas del mismo sexo en el canon jurídico, se produce en base a la colonización europea o durante el desarrollo de un marco jurídico nacional posterior, a imitación de los países occidentales. En cuanto a la condena religiosa, la consideración cambia según escuela de derecho islámico (Rowson, 2012) y, aunque es frecuente la asociación de la población homosexual

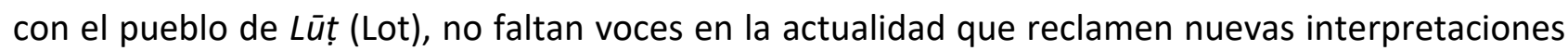
del islam (Farhat Othman, 2014; Olfa Youssef, 2008).

La introducción de la homosexualidad como delito en los códigos penales se lleva a cabo de diferente forma en función de la historia concreta de cada país, pero se puede sistematizar con arreglo a la motivación que subyace a la recogida como delito en el código penal. De este modo, el origen de la sanción puede producirse en base a: a) la doctrina legal, en general, un código legislativo de la época colonial (finales del XIX y primera mitad del s. XX) o b), en base a la doctrina religiosa.

En cuanto al primer caso, la penalización por introducción de un código legislativo colonial se puede ejemplificar con varios casos paradigmáticos, como es el de Túnez, donde el Código Penal del Protectorado francés de 1913 se mantiene vigente hasta hoy, y figura en su versión francesa la "sodomía", mientras que en la árabe se explicitan la homosexualidad tanto masculina como femenina. Lo cierto es que, tras la independencia del país en 1956, el artículo 235 no fue aplicado durante los mandatos presidenciales ni de Habib Bourghiba (1957 - 1987) ni Zine el-Abidine Ben Ali (1987 - 2011), pero sí tras la Revolución 2011, lo que en los cuatro años siguientes significó el arresto, por esta causa, de al menos cuatrocientas personas y continúa actualmente.

Asimismo, en Irak, desde 1918, se instaura el Código penal propio del Mandato británico hasta su renovación 1969, donde no se no explicita nada sobre la homosexualidad. Sin embargo, los años 1980 y 1990 son de represión hacia las personas homosexuales, llegando a instaurarse la pena capital en 2001. Tras la invasión estadounidense de 2003, y aunque se retoma el Código Penal de 1969, actualmente la homosexualidad sigue siendo condenada.

Por último, el ejemplo quizá más paradigmático sea el de Gaza, donde el Código penal del Mandato Británico sigue aún vigente desde 1936 y por ende se mantiene la sanción de las relaciones entre personas del mismo sexo.

Revista del Laboratorio Iberoamericano para el Estudio https://doi.org/10.46661/relies.6130

Sociohistórico de las Sexualidades 
En relación a la sanción por doctrina religiosa, la penalización de la homosexualidad se realiza por aplicación estricta de la legislación islámica en dos países. Son Arabia Saudí, que carece de legislación penal codificada y aplica las aleyas referidas al pueblo de Lot de la azora séptima (Q. 7:8081) e Irán, que desde 2013 aplica el código penal islámico.

\subsection{Estado actual de la legislación sobre la homosexualidad en el mundo árabe}

La situación actual en materia de legislación sobre orientación sexual en los países árabes sigue inclinando la balanza hacia la penalización de las relaciones entre personas del mismo sexo. Sin embargo, la forma de inclusión ha variado y se refieren estas prácticas de otras formas.

Con todo, como ya se ha dicho, desde los años 60 del siglo XX hay algunos países que han contemplado su despenalización. Además de los casos expuestos en el apartado anterior, la penalización de relaciones sexuales entre el mismo sexo en el mundo árabe, de acuerdo con el reciente informe de ILGA (2019), se fue llevando a cabo de manera paulatina, en algunos códigos de forma explícita como en Siria (1949), Kuwait (1960), Argelia (1966) Afganistán (1976), Yemen (1984) o Emiratos Árabes Unidos (1987).

La condena se recoge en la legislación bajo otras designaciones, por ejemplo, Libia penaliza el "coito ilícito" (1953), Marruecos legisla sobre los "delitos contra natura" y la "obscenidad" (1962), Omán incluye los "delitos ignominiosos" y "actos eróticos homosexuales o lésbicos" (1974). Mauritania recoge los "actos contra natura" (1984) y Sudán incluye la "sodomía" (1991). Asimismo, en algunos casos se recurre a formas más veladas, como son los casos de Egipto, donde se integra en la legislación en 1937, pero no se legisla explícitamente sobre homosexualidad, sino que se emplean artículos referentes a "actos escandalosos" o "incitación al libertinaje", o de Catar, donde se introdujo la homosexualidad como delito en 1971, pero se modificó su legislación al respecto en 2004. En ese momento, no se despenaliza la sodomía en sí, sino que se criminaliza a "quien incite [...] a cometer actos de sodomía o inmoralidad".

En otro orden, Líbano recoge la homosexualidad en 1943 en el Código penal, pero desde 2009 los tribunales han rechazado 4 condenas sobre la homosexualidad. Esta situación sufrió un cambio considerable en 2014, cuando se arrestó a 27 personas por esta causa.

En contrapartida, la despenalización de relaciones sexuales entre personas del mismo sexo se llevó a cabo en tres países árabes: en Jordania (1951), en el código legal de 1960; en Baréin en 1976, tras la derogación del código colonial británico y, más recientemente, en Yibuti en 1995. Con todo, la despenalización a nivel legal, no implica una aceptación dentro de la sociedad ni la ausencia de todo un imaginario sociocultural que persiste en el estigma de las personas LGTBIQ+.

\section{Encuentro entre el pensamiento musulmán y el occidental contemporáneo: un islam queer}

El acercamiento del pensamiento islámico a la teoría crítica no es nuevo ni siquiera en las sociedades árabes, donde el desarrollo del movimiento feminista se remonta a los principios del siglo XX, siendo quizá las figuras más conocidas las que conformaron el feminismo egipcio en esa época (Ruiz de Almodóvar Sel, 1989). El desarrollo del movimiento feminista no reducirá su impacto ni su crítica exclusivamente al pensamiento islámico, asumiendo algunas de las corrientes unos postulados secularistas y haciendo una crítica de la relación entre religión y Estado (Karam, 1998; Al-Ali, 2000).

De forma análoga a la evolución de los estudios feministas en Occidente, la crítica feminista ahondará, posteriormente, en la historia de las mujeres en las sociedades musulmanas, así como en las raíces históricas de la problemática de género en estas sociedades (Mernissi, 1987; Ahmed, 1992) y, del mismo modo, la transversalidad de género alcanza a la hermeneútica islámica, sea desde una aproximación ligada a la justicia social, entendida como igualdad de clase, raza y género

Revista del Laboratorio Iberoamericano para el Estudio Sociohistórico de las Sexualidades https://doi.org/10.46661/relies.6130 
(Wadud, 1995) o más directamente relacionada con la propia exégesis del texto sagrado (Wadud, 1999 y 2013; Ali, 2015).

La teoría crítica postfeminista alcanza también su desarrollo y, de este modo, los retos y los conflictos conceptuales que suponen la construcción de identidades sexogenéricas disidentes en contextos musulmanes ocupan un nuevo espacio dentro de la teoría crítica y otros espacios de la academia (Musić, 2003). De este modo, se llevan a cabo estudios sobre la cuestión homosexual (Siraj al-Haqq Kugle, 2006). Sin embargo, estos acercamientos no se limitan a esta cuestión, abordando en algunos casos el conjunto del marco teórico posmoderno y su relación con el islam (Ahmed, 2013). No faltan tampoco abordajes de la problemática que se genera en este cruce entre la interpretación religiosa y la diversidad sexual y de género desde la interseccionalidad (Rahman, 2010) o desde el marco del conflicto religión - secularismo - aceptación (Mahomed, 2016). Más aún, recientemente, se ha llevado a cabo una de las revisiones más completas de la epistemología queer musulmana desde una perspectiva anarquista (Abdou y Veneuse, 2019).

Este desenvolvimiento de la teoría posfeminista repercute también, como no podía ser de otro modo, en la hermenéutica y la exégesis coránica y, por tanto, en la consideración de aquellos aspectos relacionados con la diversidad sexual y de género en el texto sagrado (Siraj al-Haqq Kugle, 2016), planteándose la posibilidad de entender los textos islámicos como fuente de aceptación de la diversidad sexual y de género (Hendricks, 2010), atendiendo a las sentencias jurídicas sunníes (fatwas) relacionadas con la transexualidad y las cirugías reasignación de sexo (Tolino, 2017) e, incluso, contemplando la posibilidad teológica islámica de un tercer género (Alipour, 2017).

La cristalización de estas identidades musulmanas es de cariz muy heterogéneo (Siraj al-Haqq Kugle, 2010 y 2014, Rahman, 2014), por lo que no debe extrañar la cantidad de factores a tener en cuenta para un estudio riguroso, ya que no abarcan sólo el contexto geográfico (mundo árabo-islámico vs. sociedades occidentales), el origen (nacional vs. inmigrante y dentro de esta categoría el tipo de inmigración: inmigrantes de segunda o tercera generación vs. refugiados), la socialización, el grado de islamización del medio tanto familiar como social, el nivel económico, el acceso a la educación y al conocimiento de fuentes que permitan una vivencia de este islam menos ortodoxo, lo que redunda en el peso de la interseccionalidad en los procesos de construcción de ese tipo de identidades. Por ello, gran parte de los trabajos que se realizan sobre estos grupos poblacionales son monográficos y tratan la configuración de estas identidades en territorios concretos. En consecuencia, son diferentes los resultados de las investigaciones llevadas a cabo sobre contextos localizados dentro del mundo araboislámico -por ejemplo, sobre las identidades musulmanas queer en Marruecos (Sterel, 2018)- a los hallados en contextos de diáspora -por ejemplo, sobre comunidades musulmanas en el mundo occidental, como pueden ser aquellos que versan sobre la emigración sudasiática musulmana en Australia (Abraham, 2009) o en Gran Bretaña (Yip, 2004), sobre la construcción identitaria de gays musulmanes progresistas en Estados Unidos (Minwalla et alii, 2005)- o sobre la gestión de una ciudadanía sexualmente disidente y musulmana desde una perspectiva femenina (Yip, 2008).

Con todo, estas nuevas perspectivas no se han quedado exclusivamente en aportes teóricos o, desde la academia en el estudio de la configuración de identidades de grupos poblacionales concretos, sino que también se han materializado en prácticas sociales concretas. Este es el caso del matrimonio islámico entre personas del mismo sexo al que también se ha dado cabida dentro de la legislación islámica (Hendricks, 2008; El Menhayi, 2012; Jahangir y Abdullatif, 2016).

De este modo, se han logrado algunos éxitos como la celebración del primer matrimonio islámico, del que se tiene constancia a nivel público, entre personas del mismo sexo en una mezquita de Sudáfrica en 2012, oficiada por el imam y activista por los derechos de las personas LGBTIQ de origen 
argelino Ludovic Zahed. La repercusión fue tal que se continúan llevando a cabo este tipo de uniones en mezquitas en otros países como Francia desde $2013^{13}$.

La encarnación de esta intersección no solo se da en estas prácticas sociales, sino que además surgen desde hace unas décadas colectivos de musulmanes LGBTIQ+, fundamentalmente en Occidente, como en España es el más reciente caso de la red Nasij, colectivo nacido en Barcelona ${ }^{14}$.

\section{Identidades LGTBIQ+ en el mundo árabe hoy en día}

Las identidades LGTBIQ+ en el Mundo Árabe pueden ser tratadas desde varias perspectivas: la encarnación individual de estas identidades y la vehiculación de sus derechos, así como la representación social de la diversidad sexual y de género en diferentes manifestaciones culturales. Del mismo modo, no se podría dejar de lado en este apartado el posicionamiento de las instituciones ante las mismas, dada la situación de subalternidad de las personas LGTBIQ+ con la que son conniventes de diferentes formas, por ejemplo, negando su existencia, sosteniendo que se trata de una enfermedad, de una importación occidental o promoviendo y perpetuando una legislación hostil hacia estas personas.

\subsection{Encarnación de identidades LGTBIQ+ y la vehiculación de movimientos de defensa de sus derechos}

Las siglas de lesbianas, gays, transexuales, bisexuales, intersexuales, queer y otros (+) suponen la condensación en identidades culturales que permitieron la vehiculación de movimientos de reivindicación de derechos, fundamentalmente a partir de la segunda mitad del siglo XX en el mundo occidental.

En este sentido, la asunción de una identidad que se englobe dentro de las siglas LGBTIQ+ y la enunciación de sus discursos a nivel individual y grupal conlleva la representación de identidades colectivas y la vindicación de derechos.

Desde la crítica poscolonial, se plantea el problema que surge ante la asunción de estas identidades políticas, que se pueden entender como un sistema importado de occidente, argumento del que se apropian incluso instituciones que niegan la presencia de población LGTBIQ+ en el mundo árabe. La crítica poscolonial, heredera de los trabajos de Edward Said, no niega que haya habido diversidad sexual y de género, sino que la conceptualización hecha desde los estudios de género obvia los sistemas autóctonos (Massad, 2007). Todo ello redunda, por tanto, en si se trata de identidades importadas o autóctonas. Del mismo modo, hay estudios monográficos recientes que pueden centrarse en un contexto geográfico y una de las siglas que engloba la población LGTBIQ+, por ejemplo, sobre la situación de la transexualidad en el Irán contemporáneo (Najmabadi, 2014). Sin embargo, no debe extrañar la crítica de Massad, ya que debe tenerse en cuenta que gran parte de la literatura académica que trata este ámbito desde principios del s. XX, fundamentalmente desde la antropología, perpetúa hasta bien entrado el siglo XX los postulados orientalistas, que a su vez continúan un enfoque colonialista en las descripciones que llevan a cabo, es el caso, por ejemplo, del tratamiento y la conceptualización hecha, entre otros, del travestismo femenino en ciertas comunidades del sur de Iraq (Westphal-Hellbusch,1956 [trad. Rose, 1997]) o la complejidad

\footnotetext{
${ }^{13}$ https://www.saphirnews.com/Un-mariage-homosexuel-entre-musulmans-celebre-a-Sevran_a14015.html [en línea: 29-07-2021]

${ }^{14}$ Entrevista a uno de sus miembros en 2016: [ttps://www.eldiario.es/sociedad/entrevista_128_3923369.html [en línea: 07-08-2021]
}

Revista del Laboratorio Iberoamericano para el Estudio https://doi.org/10.46661/relies.6130

Sociohistórico de las Sexualidades 
epistemológica que se advierte en las sucesivas descripciones en los años 70 de janīt en Omán como homosexuales, prostitutos masculinos o como un tercer género (Murray, 1997b).

En otro orden, recientes estudios han reflejado la situación y desarrollo de colectivos, asociaciones y movimientos en defensa de los derechos del colectivo LGBTIQ+ en los países árabes (Tozcane, 2017). Asimismo, hemos realizado un análisis de la vehiculación del movimiento de reivindicación y el uso de lenguas extranjeras y vernáculas en los discursos del ciberactivismo en defensa de los derechos LGTBIQ+, que puede dar cuenta de la multiplicidad de factores implicados en este hecho (Rodríguez Iglesias, 2019b): por una lado, la necesidad de aval y protección de entidades supragubernamentales, generalmente europeas o de corte occidental, y al mismo tiempo de visibilización y enunciación de un discurso propio en el propio entorno, que permita romper la estereotipia de estigmatización tan presente en el medio sociocultural autóctono.

En este sentido, desde el propio medio, se han llevado a cabo acciones exo-grupales, desde otros colectivos que reivindican los derechos de estas personas, y endo-grupales, es decir, desde colectivos conformados por personas LGTBIQ+. Un ejemplo de las primeras serían las campañas llevadas a cabo desde colectivos de profesionales de la medicina como HELEM (ár. "sueño") en el Líbano para la concienciación social sobre la despatologización de las personas LGTBIQ+. Del segundo tipo, de acciones endo-grupales, son ejemplo la creación de colectivos tunecinos como Mawjouddine (ár. "presentes") o el específicamente lésbico Chouftouhonna (ár. "Ias ven") o la creación de asociaciones, como es el caso de la asociación tunecina SHAMS (ár. "sol"), la primera asociación en defensa de los derechos de las personas LGTBIQ+ legalizada en el mundo árabe en 2015.

\subsection{Representaciones de la diversidad sexual y de género en las manifestaciones culturales}

La literatura contemporánea comienza a introducir personajes abiertamente homosexuales a mediados del siglo XX, siendo un pionero Naguib Mahfud en su obra El callejón de los milagros (1947) quien presenta un padre de familia que mantiene relaciones homosexuales en baños públicos, temática que también recoge en otras de sus obras (Matar, 1990). Posteriormente, lo harán más autores, como Youssef Idris en su obra Abū al-Riŷāl ("El padre de los hombres") (1989), que ha sido también interpretada como una sátira a la figura de Abdel Gamal Nasser.

La representación en la literatura del amor entre mujeres en la literatura contemporánea (AlSamma, 2008) aparece más tarde. Así, la primera novela es la de la autora libanesa Imen Mansur Ana hiya enti (2000). Del mismo modo, la primera protagonista transexual de una novela será la autobiografía de la argelina Randa, ficcionalizada y editada por el editor libanés Hatem Saqiat (coa.), Mudakkirat Randa al-Trans (2010) ("Memorias de la transexual Randa").

Con todo, debe tenerse en cuenta el peso y la influencia de la colonización en el nacimiento de una Literatura Árabe LGBTIQ+, ya que desde la homosexualidad ha sido tratada en otras lenguas por autoras y autores de este origen como por ejemplo los escritores marroquíes que escriben en lengua francesa Rachid O., cuya identidad sigue oculta, Abdella Taïa o Fátima Daas.

En el cine, las narrativas de las disidencias sexuales y de género en Oriente Próximo también empiezan a tener cabida (Beirne y Habib, 2012), siendo una de las primeras apariciones de la homosexualidad la ofrece el director tunecino Nouri Bouzid, en su película Rīh al-Sadd (1986) (ár. El hombre de cenizas/ lit.: El aire de las cenizas). De interés, son también trabajos sobre la homosexualidad en el cine egipcio (Menicuci, 1998) y el análisis de la tensión que se produce en la pantalla entre esta y el colonialismo (Hassan, 2002).

La producción y reproducción de películas que expongan temática LGBTIQ+ sigue siendo una actividad que se mantiene, sea con posturas moralistas para el gran público, como por ejemplo en El Edificio Yacubián (2006), adaptación de la novela homónima del egipcio Ala Al-Aswani (2002), o 
bien mediante la producción subversiva. Por ejemplo, un cortometraje sobre travestis tunecinos ganó el Festival de Cine Franco-alemán de Túnez (2016). En cuanto a la reproducción de este tipo de cine, si bien a pequeña escala, lo cierto es que empieza a haber Festivales de Cine LGBTIQ+, como puede ser el Festival de Cinéma Queer de Mawjouddine, celebrado en 2018, en 2019 y previsiblemente en 2021.

\subsection{Posicionamiento desde las instituciones}

Una de las principales problemáticas derivadas de todo lo expuesto anteriormente es la posición que mantienen muchas instituciones, así como muchas autoridades políticas que fundamentalmente niegan de la existencia de la población LGBTIQ+ en sus países, indicando en algunos casos que se trata de un fenómeno de importación occidental. Así, la interacción entre el derecho internacional y las legislaciones islámicas conlleva una serie de tensiones (Vakulenko, 2007; Waites, 2009).

En el este sentido, más allá de hacer un estudio detallado y riguroso sobre estos casos, sirva de ejemplo el rechazo de la Organización de la Conferencia Islámica (hoy en día "para la Cooperación Islámica") a la Declaración sobre orientación sexual e identidad de género de 2007 de la Organización de Naciones Unidas. Este rechazo conllevó además la firma de una declaración contraria (54 países firmantes) ante el peligro de normalización y legitimación de "actos deplorables".

\section{Conclusiones}

El cruce entre los estudios LGBTIQ + y los estudios árabes e islámicos es un campo de estudio interseccional en pleno auge. Si bien la categoría LGBTIQ+ está motivada por una serie de acontecimientos y sucesos históricos vinculados al mundo occidental contemporáneo, lo cierto es que la diversidad sexual y de género ha estado presente en el mundo árabe e islámico, como en el resto de sociedades, desde sus orígenes. Prueba de ello son los testimonios literarios que se recogen sobre homoerotismo y prácticas sexuales entre personas del mismo sexo desde la literatura de época preislámica, la tolerancia a la fluidez en la expresión de género en ciertos contextos como los harenes o a expresiones de género no binarias que existían previamente en territorios bajo égida islámica tras la expansión del islam hacia India o el Extremo Oriente. Del mismo modo, la presencia de la diversidad sexual y de género trasciende la literatura y se rastrea desde ámbitos tan dispares como la legislación medieval islámica, que incluso promulga doctrina sobre cuestiones como la intergenitalidad, o la mística.

El cambio hacia una sociedad moderna en el contexto arabo-islámico es promovido fundamentalmente por una serie de reformas llevadas a cabo por el gobierno otomano en el siglo $\mathrm{XIX}$, lo que conlleva que se desarrollen sistemas legales a imitación de los regímenes modernos occidentales. Aunque en un principio la homosexualidad queda fuera de estos, dado que el modelo napoleónico del que bebe el modelo otomano tampoco la contemplaba, a lo largo del siglo XX se fue incluyendo en las diferentes legislaciones, ya fuera recurriendo a una justificación religiosa basada en una interpretación específica del Corán o bien, mediante una justificación legal sustentada en los posteriores códigos introducidos por el aparato colonial, fuera francés o británico. Con todo, una minoría de países de este ámbito geográfico han despenalizado las relaciones sexuales entre persona del mismo sexo.

Actualmente la persecución de carácter legal y la sanción religiosa conviven con la emergencia de movimientos sociales y referentes culturales LGBTIQ+ en el Mundo Árabe, donde las representaciones sociales se producen, ya sea de manera más o menos clandestina, y se refleja en la literatura, el cine o la música. En este sentido es importante destacar el diálogo que se da, desde hace más de un siglo entre la teoría crítica, en un principio fundamentalmente desde el feminismo, y la exégesis coránica, pues la evolución de este diálogo ha supuesto el surgimiento de nuevas 
interpretaciones, abarcando incluso la teoría queer. Es más, esta nueva exégesis ha tenido un impacto significativo entre algunas comunidades musulmanas, tanto dentro como fuera del Mundo Árabe, llegando como se ha visto a la celebración de matrimonios islámicos entre personas del mismo sexo en época reciente. 


\section{Referencias}

Abdou, M. y Veneuse, M. J. (2019). Islam and Queer Muslims: Identity and Sexuality in the Contemporary World. (Tesis doctoral). Queen's University, Ontario, editada en The Anarchist Library.

Abraham, I. (2009). "Out to Get Us": Queer Muslims and the Clash of Sexual Civilization in Australia. Contemporary Islam, 3 (1), 79-97.

Najmabadi, A. (2014). Professing Selves: Transexuality and Same-Sex Desire in Contemporary Iran. Londres, Reino Unido: Duke University Press.

Ahmed, A. S. (2013). Postmodernism and Islam: Predicament and Promise. New York, Estados Unidos de América: Taylor \& Francis.

Al-Ali, N. (2000). Secularism, Gender and the State in the Middle East: The Egyptian Women's Movement. Cambridge, Reino Unido: Cambridge University Press.

Ali, K. (2015). Sexual Ethics and Islam: Feminist Reflections on Qur'an, Hadith and Jurisprudence. Oxford, Reino Unido: Oneworld Publications.

Alipour, M. (2017). Transgender Identity, The Sex-Reassignment Surgery Fatwas and Islamic Theology of a Third Gender. Religion and Gender, 7, (2), $164-179$.

Al-Samma, H. (2008). Out of the Closet: Representation of Homosexuals and Lesbians in Modern Arabic Literature. Journal of Arabic Literature, 29 (2), 270-310.

Álvarez Morales, C. (2010). La sociedad de Al-Ándalus y la sexualidad. En Delgado Pérez, M. M./López-Anguita, G. (Eds.), Actas del Congreso Conocer Al-Andalus: perspectivas desde el siglo XXI. (pp. 43-76) Sevilla, España: Alfar.

Amer, S. (2009). Medieval Arab Lesbians and Lesbian-Like Women. Journal of the History of Sexuality, 18 (2), 215-236.

Arié, R. (1985). Ibn Hazm et l'amour courtois. Revue des mondes musulmans et de la Méditerranée: Al-Andaus - Culture et société (49), 75-89.

Beirne, R y Habib, S. (2012). Trauma and Triumph: Documenting Middle Eastern Gender and Sexual Minorities in Film and Television. En Pullen, C. (Ed.), LGBT Transnational Identity and the Media (pp. 41-58). Basingtoke, Reino Unido: Palgrave Macmillan.

Boswell, J. (1980 [trad. 1992]). Cristianismo, tolerancia social y homosexualidad. Barcelona, España: Muchnik Editores, SA.

Corriente Córdoba, F. y Monferrer Sala, J. (2005). Las diez mu'allaqat. Madrid, España: Hiperión.

Dickemann, M. (1997). "The Balkan Sworn Virgin: A Cross-Gendered Female Role”, en Murray, S. O. (Ed.), Islamic homosexualities: culture, history and literature. (pp. 197-203. New York, Estados Unidos de América: New York University Press.

El-Azhari, T. (2019) Queens, Eunuchs and Concubines in Islamic History (661-1257). Edimburgo, Reino Unido: Edinburgh University Press.

El Menyawi, H. (2012), Same-sex Marriage in Islamic Law. Wake Forest Journal of Law \& Policy, 2, 375-533.

Ferrando, I. (2001). Introducción a la historia de la lengua árabe. Nuevas perspectivas. Zaragoza, España. ISBN 10: 8492158816. 
Guardi, J. y Vanzan, A. (2012). Che genere di islam: omosessuali, queer e transessuali tra shari'a e nuove interpretazioni. Roma, Italia: Ediesse Editore.

Grémaux, R. (1993). Woman becomes Man in the Balkans. En Herdt, G. (Ed.), Third Sex Third Gender: Beyond Sexual Dimorphism in Culture and History (pp. 241-281), Nueva York, Estados Unidos de América: Zone Books.

Habib, S. (2009). Arabo-Islamic Texts on Female Homosexuality: 850-1780 AD. Nueva York, Estados Unidos de América: Teneo Press.

Habib, S. (2012). Female Homosexuality in the Middle East: Histories and Representations. Nueva York, Estados Unidos de América: Routledge.

Habib, S. (ed.) (2010). Islam and homosexuality. vol. I y II. Santa Bárbara, Estados Unidos de América: Greenwood.

Hassan, O (2002). Real Queer Arabs: The tension between Colonialism and Homosexuality in Egyptian Cinema. Film International, 8 (1), 18-24.

Hayes, J. (2000). Queer Nations: Marginal Sexualities in the Maghreb. Chicago, Estados Unidos de América: The University of Chicago Press.

Hendricks, I. M. (2008). A way forward ljtihad: A muslim perspective on same-sex marriage. En Judge, A. M. y De Waal, S. (Eds.), To have and to hold: The making of same-sex marraige in South Africa (pp. 219-227). Johannesburgo, Sudáfrica: Jacana Media.

Hendricks, I. M. (2010). Islamic texts: A source for acceptance of Queer individuals into mainstream muslim society. The Equal Rights Review, 5, 31-51.

Ibn Said al-Magribi [trad. Emilio García Gómez]. (1978). El libro de las banderas de los campeones. Barcelona, España: Seix Barral.

ILGA (2019). Homofobia de Estado 2019. Disponible en: https://ilga.org/es/ilga-lanza-informehomofobia-estado-2019

Iyer, N. (2009). Hijra/hejira. En O’Brien, J. (Ed.), Encyclopedia of Gender and Society, Vol. 1, Los Ángeles, Estados Unidos de América: SAGE.

Jahangir, J y Abdullatif, H. (2016). Islamic Law and Muslim Same-sex unions. Minneapolis, Estados Unidos de América: Lexington Books.

Jones, S.V. (2015). Ending Bacha Bazi: Boy Sex Slavery and the Responsabilty to Protect Doctrine. Indiana International \& Comparative Law Review, 25 (1), 63-78. https://doi.org/10.18060/7909.0005

Karam, A. (1998). Women, Islamism and the State: Contemporary Feminisms in the Middle East. Londres, Reino Unido: Macmillan.

Klebe, Dorit (2005). Effeminate Professional Musicians in Sources of Ottoman-Turkish Court Poetry and Music of the Eighteenth and Nineteenth Centuries. Music in Art, 30 (1-2), 97-116.

Lind van Wijngaarden, J. W. y Rani, B. (2011). Male adolescent concubinage in Peshawar, Northwestern Pakitan. Culture Health \& Sexuality, 13 (9), 1061-72 http://dx.doi.org/10.1080/13691058.2011.599863

Lucena Romero, M. A. (2021). La casuística homoerótica a través de la antología poética de alMinhāŷī: imberbes y barbipungentes. Estudios de Asia y África, 56 (2), (172), 391-404. https://doi.org/10.24201/eaa.v56i2.2665 
Mahomed, N. (2016). Queer Muslims: Between Orthodoxy, Secularism and the struggle for acceptance, Theology \& Sexuality, 22 (1-2), 57-72.

Malti-Douglas, F. (2001). Tribadism/Lesbianism and the Sexualized Body in Medieval Arabo-Islamic Narratives. En Sautman, F., Canadé, F. y Sheingorn, P. (Eds.), Same sex love and desire among women in the Middle Ages (pp-123-141). Nueva York, Estados Unidos de América: Palgrave.

Massad, J. A. (2007). Desiring Arabs. Chicago, Estados Unidos de América: University of Chicago Press.

Matar, N. I. (1990). Homosexuality in the Early Novels of Nagueeb Mahfouz. Journal of Homosexuality, 26 (4), 77-90.

Menicuci, G. (1998). Unlocking the Arab celluloid closet: Homosexuality in Egyptian Film. Middle East Report, 206, 32-36.

Mernissi, F. (1987). L'harem politique. Paris, Francia: Albin Michel.

Milevska, S. (2005). Gender difference in the Balkans. (Tesis doctoral). University of London, Londres, Reino Unido.

Minwalla, O., Rosser, B., Simon, R., Feldan, J. y Varga, C. (2005). Identity Experience among Progressive Gay Muslims in North America: A Qualitative Study within al-Fatiha. Culture, Health and Sexuality, 7 (2), 113-28.

Mposo, P. S. (2017). Alternative Sexualities in India. (Tesis de Máster). Universidad de Leiden, Leiden, Países Bajos.

Murray, S. O. (1997a). Male Homosexuality, Inheritance Rules and the Status of Women in Medieval Egypt: The Case of Mamluks. En Murray, S. O. (Ed.), Islamic homosexualities: culture, history and literature (pp. 161-173). Nueva York, Estados Unidos de América: New York University Press.

Murray, S. O. (1997b). The Sohari Khanith. En Murray, S. O. (Ed.), Islamic homosexualities: culture, history and literature (pp. 244-255). Nueva York, Estados Unidos de América: New York University Press.

Murray, S.O. (2007). Homosexuality in the Ottoman Empire, Historical Reflections / Réflexions Historiques, 33 (19), Eighteen-Century Homosexuality in Global Perspective, 101-116.

Othman, F. (2014). Ces tabous qui défigurent l'islam. Tome II: L'apostasie et l'homosexualité. París, Francia: L'Harmattan.

Rahman, M. (2010). Queer as Intersectionality: Theorizing Gay Muslim Identities. Sociology, 44 (5), 944-961.

Rahman, M. (2014). Homosexualities, Muslim cultures and modernity. Londres, Reino Unido: Palgrave McMillan.

Rodríguez Iglesias, A. (2019a). Más allá del homoerotismo en al-Ándalus: ¿ Un caso de homoerotismo en El Collar de la Paloma de Ibn Hazm de Córdoba? En Martínez Pleguezuelos, A., Fernández Cano, M., Pérez Bernabeu, A., Sánchez Ibáñez, I. y Fernández de Pablo, S., MariCorners. Estudios interdisplinares LGTBIQ+. Madrid, España: Escuela Técnica Superior de Arquitectura de Madrid ETSAM UPM.

Rodríguez Iglesias, A. (2019b). Ciberactivismo LGBTIQ+ en el Mundo Árabe: el uso estratégico del árabe dialectal o la política lingüística como elemento identitario. Methaodos. Revisa de Ciencias Sociales, 7 (1), 74-89. http://dx.doi.org/10.17502/m.rcs.v7i1.277 
Roth, N. (1995). Hebrew Poetry. En Wilhelm, J. E. (Ed.) Gay and lesbian poetry: an anthology from Sappho to Michaelangelo (pp. 235-260). Nueva York, Estados Unidos de América: Garland Publishing Inc.

Rowson, E. (1991). The Effeminates of Early Medina. Journal of the American Oriental Society, 111 (4), 617-693.

Rowson, E. (2006). Arabic: middles ages to nineteenth century. En Brulotte, G y Phillips, J. (Eds.) Encyclopedia of erotic literature, vol. 1. Nueva York, Estados Unidos de América: Routledge.

Rowson, E. (2012). “Homosexuality in Islamic Law” en Encyclopaedia Iranica, XII, (4), pp. 441-443. Disponible en: http://www.iranicaonline.org/articles/homosexuality-ii [En línea 12-11-21]

Rowson, E. y Wright (Eds.) (1997). Homoeroticism in Classical Arabic Literature. Nueva York, Estados Unidos de América: Columbia University Press.

Ruiz de Almodóvar Sel, C. (1989). Historia del movimiento feminista egipcio. Granada, España: Feminae. Universidad de Granada. Seminario de Estudios de la Mujer.

Sanders, P. (1991). Gendering the Ungendered Body: Hermaphrodites in Medieval Islamic Law. En Keddie, N. y Baron, B. (Ed.), Women in Middle Eastern History: Shifting Boundaries in Sex and Gender, (pp. 74-96). New Heaven, Estados Unidos de América: Yale University Press.

Schuyler, E. (1876). Turkistan: Notes of a Journey in Russian Turkistan, Khokand, Bukhara and Kudja. Londres, Reino Unido: Sampson, Low, Marston, Searle \& Rivington, vol. 1.

Shafik, A. (2020). El homoerotismo masculino en la literatura mameluca: al-Mutayyam de Ibn Dānīyā". Miscelánea de Estudios Árabes y Hebreos, Sección Árabe-Islam [1696-5868] 70, 381-407. https://doi.org/10.30827/meaharabe.v70i0.15115

Siraj al-Haqq Kugle, S. (2006). On Being Homosexual and Muslims: conflicts and challenge. En Ouzgane, L. (Ed.), Islamic Masculinities (pp. 202-216), Londres, Reino Unido: Zed Books.

Siraj al Haqq Kugle, S. (2010). Homosexuality in Islam: Critical Reflection on Gay, Lesbian and Transgender Muslims. Oxford, Reino Unido: Oneworld Publications.

Siraj al Haqq Kugle, S. (2014). Living Out Islam: Voices of Gay, Lesbian and Transgender Muslims. Nueva York, Estados Unidos de América: New York University Press.

Siraj al Haqq Kugle, S. (2016). Alternative Realities: Queer Muslims and the Qur'an”. Theology \& Sexuality, 22 (1-2), 80-101.

Sterel, V. (2018). Queer Islamic Masculinities: Social and Individual Identity in Queer Moroccan Muslim Men. (Tesis de máster) Leiden University, Leiden, Países Bajos.

Tolino, S. (2017). Transgenderism, Transsexuality and Sex-reasignment Surgery in Contemporary Sunni Fatwas. Islamic Law, 17, 223-246.

Tozcane, L. (2017). Être homophobe n'est pas révolutionnaire. Europe Solidaire Sans Frontières. Disponible en web: https://www.europe-solidaire.org/spip.php?article40519.

Vakulenko, K. (2007). "Islamic Dress Headscarves" and the European Convention on Human Rights: An Intersectional Perspective. Social and Legal Studies, 16 (2), 183-99.

Veglinson, J. (1997). La poesía árabe clásica. Madrid, España: Hiperión.

Vicente, Á. (2008). Génesis y clasificación de los dialectos neo-arabes. Madrid, España: IEOP.

Wadud, A. (1995). Towards a Qur'anic Hermeneutics of Social Justice: Race, Class and Gender. Journal of Law and Religion, 12 (1), 37-50. 
Wadud, A. (1999). Qur'an and Woman: Rereading the Sacred Text from a Woman's perspective. Oxford, Reino Unido: Oxford University Press.

Wadud, A. (2013). Inside the Gender Jihad: Women's Reform in Islam. Londres, Reino Unido: Oneworld Publications.

Waites, M. (2009). Critique of "Sexual Orientation" and "Gender Identity" in Human Rights Discourse: Global Queer Politics beyond the Yogyakarta Principles. Contemporary Politics 15 (1), 137-56.

Westhal-Hellbusch, S. (1956) [trad. Rose, B., 1997]. Institutionalized Gender-Crossing in Southern Iraq. En Murray, S. O. (Ed.), Islamic homosexualities: culture, history and literature (pp. 233-243). Nueva York, Estados Unidos de América: New York University Press.

Yip, A.K.-T. (2004). Embracing Allah and Sexuality? South Asian Non-Heterosexual Muslims in Britain. En Jacobsen, K. A. y Pratap Jumar, P. (Eds.), South Asians in the Diaspora (pp. 294-310). Leiden, Países Bajos: Brill

Yip, A.K.-T. (2008). The Quest for Intimate/Sexual Citizenship: Lived Experiences of Lesbian and Bisexual Muslim Women, Contemporary Islam, 2 (2), 99-117.

حيرة مسلمة في الميراث والزواج والجنسية المثلية. دار سحر للنشر:(2008) Youssef, O

Ze'evi, Dror. (2006). Producing Desire: Changing Sexual Discourse in the Ottoman Middle East, 15001900. Berkeley, Estados Unidos de América: University of California Press. 